TRANSACTIONS OF THE

AMERICAN MATHEMATICAL SOCIETY

Volume 178, April 1973

\title{
ADDITIVE SET FUNCTIONS ON LATTICES OF SETS $\left(^{1}\right)$
}

BY

\author{
GENE A. DE BOTH( $\left.{ }^{2}\right)$
}

\begin{abstract}
This paper is concerned with properties of additive set functions defined on lattices of sets. Extensions of results of Brunk and Johansen, Darst, Johansen, and Uhl are obtained. Two fundamental approximation properties for lattices of sets (established in another paper) permit us to translate the setting and consider countably additive set functions defined on sigma lattices of sets. Thereby results for countably additive set functions defined on sigma lattices of sets are used to obtain alternate derivations and extensions of Darst's results for additive set functions defined on lattices of sets, i.e., we consider the Radon. Nikodym derivative, conditional expectation, and martingale convergence for lattices of sets.
\end{abstract}

I. Introduction. In this paper we consider additive set functions defined on lattices of sets. We shall extend results of Brunk and Johansen, Darst, Johansen, and Uhl, obtained in [2], [3], [8], and [14]. The two approximation theorems for lattices of sets obtained in [5] permit us to translate the setting ( $\$$ III) and consider $\sigma$-additive set functions defined on $\sigma$-lattices of sets. Thereby we are able to use the results obtained in [4] and results of Johansen for $\sigma$-additive set functions defined on $\sigma$-lattices of sets to obtain alternate derivations and extensions of Darst's results for additive set functions defined on lattices of sets, i.e., we discuss the Radon-Nikodym derivative ( $\S \mathrm{IV}$ ), conditional expectation $(\S \mathrm{V}$ ), and martingale convergence $(\S \mathrm{VI})$ in this setting. We state our results in the setting of the Orlicz spaces $L^{\Phi}(\Omega, \mathcal{U}, \mu, R)$ and $V^{\Phi}(\Omega, \mathcal{U}, \mu, R)$ as defined by Uhl in [14], where $\mathcal{U}$ is an algebra of subsets of $\Omega$ and we assume that $\mu$ is a nonnegative bounded additive function on $U$. In $\$$ VII we extend our results to include vector valued $\left(R^{n}\right)$ set functions.

Received by the editors August 3, 1971 and, in revised form, April 27, 1972.

AMS (MOS) subject classifications (1970). Primary 02J05, 28A05, 28A10, 28A15, 28A20, 46E 30, 60G45.

Key words and phrases. Lattice of sets, algebra of sets, finitely additive set functions, sigma algebra, sigma lattice, $\Delta_{2}$-condition, Orlicz space, Radon-Nikodym derivative, conditional expectation, martingale.

(1) This paper contains some of the main results of the author's doctoral dissertation written at Purdue University under the guidance of Professor Richard B. Darst to whom the author expresses his sincerest thanks.

(2) The author was supported by a National Science Foundation Science Faculty Fellowship: NSF grant no. 60045. 
II. Preliminaries. We begin by recalling some basic definitions.

Definition 2.1. A class $\pi$ of subsets of a nonempty set $\Omega$ is called a lattice of subsets of $\Omega$ if both the empty set $\varnothing$ and $\Omega$ belong to $\mathbb{M}$ and both $A \cup B$ and $A \cap B$ are in $M$ whenever $A$ and $B$ are elements of $M$. If in addition $M$ is closed under countably infinite unions and intersections, then $M$ is called a $\sigma$-lattice.

For example, in a metric space both the class of open sets and the class of closed sets are lattices of sets.

Definition 2.2. A real valued set function $\mu$ on $\mathbb{N}$ is said to be additive if

$$
\begin{aligned}
\mu(\varnothing) & =0 \text { and } \\
\mu(A \cup B)+\mu(A \cap B) & =\mu(A)+\mu(B), \quad A, B \in \mathbb{M} .
\end{aligned}
$$

The function $\mu$ is said to be $\sigma$-additive (countably additive) if, in addition, for $A_{i} \cap A_{j}=\varnothing, i \neq j$,

$$
\mu\left(\bigcup_{n=1}^{\infty} A_{n}\right)=\sum_{n=1}^{\infty} \mu\left(A_{n}\right), \quad A_{n} \in \mathbb{M}, n=1,2, \ldots
$$

Throughout this paper, M will denote a lattice of subsets of a nonempty set $\Omega$.

Suppose each of $\lambda$ and $\mu$ is a bounded, additive function on $M$ and $\mu$ is nonnegative (i.e. $\mu(A) \leq \mu(B)$ if $A \subset B$ ).

Results of B. J. Pettis [11, Corollary 1.2.1, p. 189] assert that a real valued function $F$ defined on a lattice $M$ has a unique additive extension to the algebra $\mathbb{Q}$ generated by $\mathbb{N}$ if, and only if, $F$ satisfies (2.2.1) and (2.2.2). Thus, each of $\lambda$ and $\mu$ has a unique additive extension to $\mathcal{Q}$. These extensions will also be denoted by $\lambda$ and $\mu$. Moreover, the conditions (2.2.1) and

$$
\mu(A \cup B)=\mu(A)+\mu(B), \quad A, B \in \mathbb{M}, A \cap B=\varnothing,
$$

do not imply (2.2.2).

Definition 2.3. An extended real valued function $f$ on $\Omega$ is said to be $\pi$ measurable if $[f>a] \subseteq M, a \in R$; if the range of $f$ is a finite set then $f$ is called a simple function.

For example, the bounded upper semicontinuous functions on $I=[0,1]$ are uniform limits of simple upper semicontinuous functions which are measurable with respect to the lattice of closed subsets of $I$.

Suppose that $\mathbb{N}$ is a sublattice of an algebra $\mathcal{U}$ of subsets of $\Omega$ and that $\mu$ is a nonnegative bounded additive function on $\mathrm{U}$.

In order to state the results in the following sections succinctly, we recall some basic facts about Orlicz spaces. Suppose that $\Phi$ is a continuous Young's function $[14, \mathrm{p} .20]$ satisfying the $\Delta_{2}$-condition ( $\Phi$ satisfies the $\Delta_{2}$-condition if there exists $0<K<\infty$ such that $\Phi(2 x) \leq K \Phi(x)$ for all $x \in R)$. Then $\Phi$ is an 
even, convex function defined on the real numbers $R$ with $\Phi(0)=0$ and $\Phi(x) \neq 0$ for some $x$. Examples are $\Phi(x)=|x|^{p}, 1 \leq p<\infty$.

The Orlicz space $L^{\Phi}(\Omega, \mathcal{U}, \mu, B), B$ a Banach space, is the space of equivalence classes of totally $\mu$-measurable functions $f$ on $\Omega$ such that

$$
\int_{\mathbf{\Omega}} \Phi(\|f(x)\|) d \mu<\infty
$$

where totally $\mu$-measurable and integration are as in Chapter III of Dunford and Schwartz [6]. By [9, Theorem 9.4, p. 76], if $\Phi$ satisfies the $\Delta_{2}$-condition, and $B=R$, then convergence in norm means

$$
\lim _{n \rightarrow \infty} \int_{\mathbf{Q}} \phi\left(\left\|f(x)-f_{n}(x)\right\|\right) d \mu=0
$$

The completion $V^{\Phi}(\Omega, \mathcal{U}, \mu, B)$ of $L^{\Phi}(\Omega, \mathcal{U}, \mu, B)$ is a space of $\mu$-continuous additive set functions on $\mathcal{U}$. Information about these $L^{\Phi}$ and $V^{\Phi}$ spaces can be found in [14] and the references therein. By [14, Corollary 7, p. 49], step functions (indefinite integrals of simple functions) are dense in $V^{\Phi}$. Hence $H \epsilon$ $V^{\Phi}$ can be identified with a sequence $\left\{b_{n}\right\}_{n=1}^{\infty}$ of simple $\mathcal{U}$-measurable functions and

$$
\|H\|_{\Phi}=\operatorname{Lim}_{n \rightarrow \infty}\left\|b_{n}\right\|_{\Phi}
$$

If $\Phi(x)=|x|^{p}, p \geq 1$, then we denote $L^{\Phi}$ by $L_{p}$ and $V^{\Phi}$ by $V_{p}$. Since $\mu$ is finite, $L^{\Phi} \subset L_{1}, V^{\Phi} \subset V_{1}$, and

$$
\|\cdot\|_{1} \leq M\|\cdot\|_{\Phi} \cdot
$$

III. Applying the isomorphisms $\tau, T$, and $U$ to translate the selting. Suppose each of $\lambda$ and $\mu$ is a (real valued) bounded, additive function on $\pi$ and $\mu$ is nonnegative, and that $\mathscr{A}$ is the algebra generated by $\mathbb{M}$. Recall that we are also denoting the unique extensions of $\lambda$ and $\mu$ to $\mathbb{Q}$ by $\lambda$ and $\mu$.

Referring the reader to Pp. 97, 164-167,240,274-276, and $311-313$ of Dunford and Schwartz [6] for details, we recall that there is a totally disconnected compact Hausdorff space $\Omega_{1}$ and an isomorphism $\tau$ of $\mathbb{Q}$ onto the algebra $\mathbb{C}_{1}$ of closed and open subsets of $\Omega_{1}$ such that the images $\lambda_{1}$ and $\mu_{1}$ of $\lambda$ and $\mu$ (i.e. $\left.\mu_{1}(\tau(A))=\mu(A)\right)$ are countably additive on $\mathbb{Q}_{1}$. Consequently, $\lambda_{1}$ and $\mu_{1}$ have unique countably additive extensions $\lambda_{2}$ and $\mu_{2}$ to the $\sigma$-algebra $\mathfrak{Q}_{2}$ generated by $\mathfrak{Q}_{1}$. The mappings $T: \mu \rightarrow \mu_{1}$ and $U: \mu_{1} \rightarrow \mu_{2}$ are isometric isomorphisms.

Let $\mathbb{M}_{1}=\tau(\mathbb{M})=\left\{A_{1}: A_{1}=\tau(A), A \in \mathbb{M}\right.$. Then $\mathbb{M}_{1}$ is a lattice of subsets of $\Omega_{1}$ and the algebra $Q_{1}$ is the algebra generated by $\mathbb{M}_{1}$. The lattice $\mathbb{M}_{1}$ generates a $\sigma$-lattice $\mathbb{M}_{2}$ which is contained in the $\sigma$-algebra $\mathfrak{Q}_{2}$. But $\lambda_{2}$ and $\mu_{2}$ are countably additive on $\mathbb{Q}_{2}$, and hence on $\mathbb{M}_{2}$.

The approximation theorems for lattices of sets established in [5] $\left(\pi_{1}\right.$ is dense in $\pi_{2}$ and $\pi_{2}$-measurable functions can be approximated by simple $\pi_{1}$ - 
measurable functions in $\left.L^{\Phi}\left(\Omega_{1}, \mathbb{Q}_{2}, \mu_{2}, R\right)\right)$ and the isomorphisms $\tau, T$, and $U$ are used in the following sections to obtain results for $\lambda$ and $\mu$ on $M$ from the corresponding results for $\lambda_{2}$ and $\mu_{2}$ on $\mathbb{M}_{2}$. Uhl [14] used these isomorphisms to obtain the derivative of $\lambda$ with respect to $\mu$ on $\mathfrak{Q}$.

IV. The Radon-Nikodym derivative given a lattice of sets. We are considering real valued bounded additive set functions, $\lambda$ and $\mu$, defined on a lattice $\pi$ of sets. The following example illustrates the fact that even if $\lambda$ and $\mu$ are countably additive, $M$ is a $\sigma$-lattice, and $\lambda$ is absolutely continuous with respect to $\mu$, it may not be possible to approximate $\lambda$ by integrals of simple M-measurable functions. Recall that $f$ is $\mathbb{M}$-measurable if $[f>a] \in \mathbb{M}$ for all $a \in R$.

Example 4.1. Suppose $\Omega=\{a, b\}, \mathbb{M}=\{\varnothing,\{a\}, \Omega\}, \mathscr{A}=\{\varnothing,\{a\},\{b\}, \Omega\}$, $\lambda(\varnothing)=0, \lambda(\{a\})=2, \lambda(\{b\})=4, \lambda(\Omega)=6, \mu(\varnothing)=0, \mu(\{a\})=\mu(\{b\})=1$, and $\mu(\Omega)=2$. Then for $f$ to be $\mathbb{N}$-measurable, $f(a) \geq f(b)$, because if $f(a)<c<f(b)$, then $[f>c]=\{b\} \notin \mathbb{M}$. Therefore, if $F(E)=\int_{E} f d \mu$, where $f$ is a simple $\mathbb{M}$-measurable function, then $F(\{a\}) \geq F(\{b\})$. But $\lambda(\{a\})=\lambda(\{b\})-2$, thus it is impossible to approximate $\lambda$ by integrals of simple $\mathbb{M}$-measurable functions. Moreover, suppose $f(a)=2$ and $f(b)=-3$; then $f=2 \times\{a\}+(-3) \chi\{b\}$. Hence, $f$ is a simple $\mathbb{M}$-measurable function, but we cannot write $f=\Sigma x_{n} \chi_{E_{n}}$, where $E_{n} \in \mathbb{M}$ and $E_{n}$ is the set on which $f$ takes on the value $x_{n}$. Notice also that the functions $-f,|f|$, and $f^{2}$ are not $M$-measurable.

Nevertheless, Radon-Nikodym derivatives have been defined for additive set functions on lattices of sets.

In [8], S. Johansen defined, constructed, and established the uniqueness of a Randon-Nikodym derivative for $\sigma$-additive set functions defined on $\sigma$-lattices of sets. We shall obtain information about $\lambda$ and $\mu$ by applying Johansen's results to $\lambda_{2}$ and $\mu_{2}$ (where $\lambda, \mu, \lambda_{2}$, and $\mu_{2}$ are as in $\S$ III).

In [3], R. B. Darst established the analogous definition, construction, and uniqueness of a Radon-Nikodym derivative for nonnegative, bounded, finitely additive set functions defined on lattices of sets. For the sake of completeness, we state the definition for the case where $\lambda$ is not assumed to be nonnegative. The appearance of $M$ in the definition is a technical inconvenience made necessary by the fact that $\left[f_{n} \leq b\right]=\Omega$ and $\left[f_{n}>a\right]=\Omega$ for fixed $n$ and large positive $b$ and large negative $a$.

Definition 4.2. We call a sequence $\left\{f_{n}\right\}$ of simple M-measurable functions defined on $\Omega$ a derivative (or a "sequence of approximate derivatives") of $\lambda$ with respect to $\mu$ on $\mathbb{R}$ if $\left\{f_{n}\right\}$ is a $L_{1}$-Cauchy sequence: $\int_{\Omega}\left|f_{m}-f_{n}\right| d \mu \rightarrow 0$, and, for every $\epsilon>0, M>0$, there is a positive integer $N$ such that if $n>N$, $a, b \in R, b<M, a>-M$, and $A, B \in \mathbb{M}$, then

$$
\lambda\left(A \cap\left[f_{n} \leq b\right]\right)<b \mu\left(A \cap\left[f_{n} \leq b\right]\right)+\epsilon,
$$




$$
\lambda\left(B^{C} \cap\left[f_{n}>a\right]\right)>a \mu\left(B^{C} \cap\left[f_{n}>a\right]\right)-\epsilon .
$$

In Example 4.1, the function $f$ defined by $f(a)=f(b)=3$ is the Radon-Nikodym derivative of $\lambda$ with respect to $\mu$ on $M$. However, the function $g$ defined by $g(a)$ $=2$ and $g(b)=4$ is the classical Radon-Nikodym derivative of $\lambda$ with respect to $\mu$, and $f \notin g$ everywhere. The function $g$ is not $\mathbb{M}$-measurable.

We now turn to the construction of the Radon-Nikodym derivative of $\lambda$ with respect to $\mu$ on $\mathbb{M}$. We assume that both $\lambda$ and $\mu$ are nonnegative.

Suppose we let $\Phi(x)=|x|, x \in R$. Then $L=L_{1}$, and Johansen's derivative $f \in L_{1}$ of $\lambda_{2}$ with respect to $\mu_{2}$ on $\Pi_{2}$ can be approximated [5, Theorem 2] by a $L_{1}$-Cauchy sequence $\left\{f_{n}^{\prime \prime}\right\}$ of simple $\mathbb{M}_{1}$-measurable functions. In the proof of $\left[5\right.$, Theorem 2], the interval $[0, n]$ is split into $n 2^{n}$ equal parts of lengths $1 / 2^{n}$ and then the simple $\mathbb{M}_{1}$-measurable function $f_{n}^{\prime \prime}$ is defined by

$$
f_{n}^{\prime \prime}= \begin{cases}n & \text { on } E_{0}, \\ n-j / 2^{n} & \text { on } E_{j} \cap\left(\bigcup_{i=0}^{i-1} E_{i}\right)^{c}, \quad j=1, \ldots, n 2^{n},\end{cases}
$$

where $E_{j} \in \mathbb{M}_{1}$ (applying [5, Theorem 1]) is close to $\left[/>n-j / 2^{n}\right], j=0,1, \ldots$, $n 2^{n}-1$, and $E_{n 2^{n}}$ is close to $[f \geq 0]$, in measure (we can use $\lambda_{2}+\mu_{2}$ instead of $\left.\mu_{2}\right)$. If we let $r\left(A_{j}\right)=E_{j}$, then by the inverse image of $f_{n}^{\prime \prime}$, under $\tau$, we mean the simple M-measurable function $f_{n}$ defined by

$$
f_{n}= \begin{cases}n & \text { on } A_{0}, \\ n-j / 2^{n} & \text { on } A_{j} \cap\left(\bigcup_{i=0}^{i-1} A_{i}\right)^{c}, \quad j=1, \ldots, n 2^{n} .\end{cases}
$$

Since $\mu\left(A_{j}\right)=\mu_{1}\left(\tau\left(A_{j}\right)\right)=\mu_{1}\left(E_{j}\right)=\mu_{2}\left(E_{j}\right)$, the functions $\left|f_{n}-f_{m}\right|$ and $\left|f_{n}^{\prime \prime}-f_{m}^{\prime \prime}\right|$ are finitely valued and have the same values on sets of the same measure. Consequently,

$$
\int_{\Omega}\left|f_{n}-f_{m}\right| d \mu=\int_{\Omega_{1}}\left|f_{n}^{\prime \prime}-f_{m}^{\prime \prime}\right| d \mu_{2} .
$$

Therefore $\left\{f_{n}\right\}$ is a $L_{1}$-Cauchy sequence of simple $\mathbb{M}$-measurable functions, and is, in fact, the Radon-Nikodym derivative of $\lambda$ with respect to $\mu$ on $\mathbb{M}$ (i.e., $\left\{f_{n}\right\}$ satisfies the requirements of Definition 4.2). on $M$.

Theorem 4.3. The sequence $\left\{f_{n}\right\}$ is the derivative of $\lambda$ with respect to $\mu$

Proof. First show that the sequence $\left\{f_{n}^{\prime \prime}\right\}$ satisfies the requirements of Definition 4.2 for $\lambda_{2}$ and $\mu_{2}$ on $\mathbb{M}_{2}$. We omit computations. Then to verify that the sequence $\left\{f_{n}\right\}$ satisfies (4.2.1), simply note that

$$
\begin{aligned}
\lambda(A \cap & {\left.\left[f_{n} \leq b\right]\right)=\lambda_{2}\left(\tau A \cap\left[f_{n}^{\prime \prime} \leq b\right]\right) } \\
& \leq b \mu_{2}\left(\tau A \cap\left[f_{n}^{\prime \prime} \leq b\right]\right)+\epsilon=b \mu\left(A \cap\left[f_{n} \leq b\right]\right)+\epsilon .
\end{aligned}
$$


Similarly, the sequence $\left\{f_{n}\right\}$ satisfies (4.2.2). Thus $\left\{f_{n}\right\}$ is the Radon-Nikodym derivative of $\lambda$ with respect to $\mu$ on $\pi$.

V. Conditional expectation given a lattice of sets. Recall that if $b \epsilon$ $L_{1}(\Omega, \mathcal{A}, \mu)$, where $(\Omega, \mathcal{Q}, \mu)$ is a probability space, and $\mathcal{B}$ is a sub- $\sigma$-algebra of the $\sigma$-algebra $\mathfrak{Q}$, then $f=E(b, \Re)$ is the conditional expectation of $b$ given $\mathcal{B}$ if $f$ is $\mathfrak{B}$-measurable and $\int_{A} f d \mu=\int_{A} b d \mu$ for all $A \in \mathfrak{B}$. If $b \in L_{2}(\Omega, \mathbb{A}, \mu)$, then $f \in L_{2}(\Omega, \mathbb{Q}, \mu)$ and $f$ minimizes the expression $\int_{\Omega}(b-k)^{2} d \mu$, where $k$ is $\mathbb{B}$ -

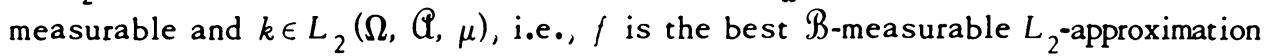
to $b$.

H. D. Brunk defined conditional expectation given a $\sigma$-lattice, $\mathbb{M}_{2}$, and established several of its properties in [1]. Denote the conditional expectation of $b \in$ $L_{1}$ given $\mathbb{M}_{2}$ by $E\left(b, \pi_{2}\right)$. For $b \in L_{2}$, Brunk characterized $E\left(b, \pi_{2}\right)$ as the unique $\Pi_{2}$-measurable best $L_{2}$-approximation to $b$, and discussed applications in which $E\left(b, \mathbb{M}_{2}\right)$ is the solution to maximum likelihood estimation problems. Subsequently S. Johansen [8] described a Radon-Nikodym derivative given a $\sigma$-lattice and showed (Theorem 5.6) that the Radon-Nikodym derivative was the conditional expectation described by Brunk in the appropriate case $\left(L_{2}\right)$. Similarly, R. B. Darst [3] showed (Theorem 5.5) that his Radon-Nikodym derivative given a lattice $M$ (Definition 4.2) was the best $\mathbb{M}$-measurable approximation in the appropriate case $\left(V_{2}\right)$. Using the isomorphisms $\tau, T$, and $U$, and the second approximation theorem for lattices, [5, Theorem 2], and applying Johansen's result (Theorem 5.6) for $\sigma$-lattices, we are able to obtain a much more transparent proof of Darst's result (Theorem 5.5) for lattices. We first show that the derivative of a $V^{\Phi}$-function is a $V^{\Phi}$-function.

Definition 5.1. If $\lambda$ is the restriction to $\mathbb{M}$ of an element $H=\left\{b_{n}\right\}_{n=1}^{\infty}$ of $V^{\Phi}(\Omega, U, \mu, R)$, then we shall denote the Radon-Nikodym derivative (Definition 4.2), $F=\left\{f_{n}\right\}_{n=1}^{\infty}$, of $\lambda$ with respect to $\mu$ on $\mathbb{M}$ by $F=E(H, \mathbb{M})$.

Definition 5.2. If $b \in L^{\Phi}\left(\Omega_{1}, \mathcal{U}_{2}, \mu_{2}, R\right)$ and $H_{2}(E)=\int_{E} b d \mu_{2}, E \in \mathcal{U}_{2}$, then we shall denote the derivative [8] $f$ of $H_{2}$ with respect to $\mu_{2}$ on $\pi_{2}$ by $f=$ $E\left(b, M_{2}\right)$.

The following theorem for $\sigma$-lattices is established in [4].

Theorem 5.3. The Radon-Nikodym derivative of an $L^{\Phi}$-function $b$ is an $L^{\Phi}$. function $f=E\left(b, \mathbb{M}_{2}\right)$.

Theorem 5.4. The Radon-Nikodym derivative of a $V^{\Phi}$-function $H$ is a $V^{\Phi}$. function $F=E(H, M)$. and $U$,

Proof. Suppose $H=\left\{b_{n}\right\}$ is in $V^{\Phi}(\Omega, \mathcal{U}, \mu, R)$. By the isomorphisms $\lambda, T$,

$$
\begin{aligned}
& \mathrm{U} \rightarrow \mathrm{U}_{1} \rightarrow \mathrm{U}_{2}, \quad \mathbb{Q} \rightarrow \mathbb{Q}_{1} \rightarrow \mathbb{Q}_{2}, \quad \Re \rightarrow \mathbb{M}_{1} \rightarrow \mathbb{M}_{2}, \\
& H \rightarrow H_{1} \rightarrow H_{2}, \quad \mu \rightarrow \mu_{1} \rightarrow \mu_{2} .
\end{aligned}
$$


If $b_{n}=\Sigma a_{k} \chi_{E_{k}}$ let $b_{n}^{\prime \prime}=b_{n}^{\prime}=\Sigma a_{k} X_{\tau} E_{k}$. Then, since $H(E)=\lim _{n \rightarrow \infty} \int_{E} b_{n} d \mu$ uniformly for all $E \in \mathcal{U}$, and $H_{1}(\tau E)=H(E)$, it follows that

$$
H_{1}(E)=\lim _{n \rightarrow \infty} \int_{E} b_{n}^{i} d \mu_{1} \quad \text { uniformly for all } E \in \mathbb{U}_{1} \text {. }
$$

Setting $\gamma_{n}(E)=\int_{E} b_{n} d \mu$ on $\mathcal{U}, \gamma_{n}^{\prime}(E)=\int_{E} b_{n}^{\prime} d \mu_{1}$ on $\mathcal{U}_{1}$, and $\gamma_{n}^{\prime \prime}(E)=\int_{E} b_{n}^{\prime \prime} d \mu_{2}$ on $\mathcal{U}_{2}$, we get

$$
\left\|H_{2}-\gamma_{n}^{\prime \prime}\right\|_{1}=\left\|H_{1}-\gamma_{n}^{\prime \prime}\right\|_{1}=\left\|H-\gamma_{n}\right\|_{1} \rightarrow 0
$$

because $U$ and $T$ are isometric isomorphisms. Thus,

$$
H_{2}(E)=\lim _{n \rightarrow \infty} \int_{E} b_{n}^{\prime \prime} d \mu_{2} \quad \text { uniformly for all } E \in \mathcal{U}_{2} \text {. }
$$

Since $\int_{\Omega_{1}} \Phi\left(b_{n}^{\prime \prime}-b_{m}^{\prime \prime}\right) d \mu_{2}=\int_{\Omega} \Phi\left(b_{n}-b_{m}\right) d \mu \rightarrow 0$, and the space $L^{\Phi}\left(\Omega_{1}, \mathcal{U}_{2}, \mu_{2}, R\right)$ is complete, there is a unique $b \in L^{\Phi}\left(\Omega_{1}, U_{2}, \mu_{2}, R\right)$ such that $b_{n}^{\prime \prime}$ converges to $b$ in $L^{\Phi}$-norm. But $\left\|b_{n}^{\prime \prime}-b\right\|_{1} \leq M\left\|b_{n}^{\prime \prime}-b\right\|_{\Phi}$; therefore

$$
\int_{E} b d \mu_{2}=\lim _{n \rightarrow \infty} \int_{E} b_{n}^{\prime \prime} d \mu_{2} \quad \text { uniformly for all } E \in \mathbb{U}_{2} \text {. }
$$

It follows from (5.4.1) and (5.4.2) that

$$
H_{2}(E)=\int_{E} b d \mu_{2} \text { for all } E \in \mathcal{U}_{2} \text {. }
$$

By Theorem 5.3, $f=E\left(b, M_{2}\right)$ is in $L^{\Phi}\left(\Omega_{1}, U_{2}, \mu_{2}, R\right)$. Thus, by [5, Theorem 2], there exists a sequence $\left\{f_{n}^{\prime \prime}\right\}$ of simple $\mathbb{M}_{1}$-measurable functions such that $\lim _{n \rightarrow \infty} \int_{\Omega_{1}} \Phi\left(f-f_{n}^{\prime \prime}\right) d \mu_{2}=0$ (which implies $\lim _{n \rightarrow \infty} \int_{\Omega_{1}}\left|f-f_{n}^{\prime \prime}\right| d \mu_{2}=0$ ). Then the inverse image under $\tau$ of the sequence $\left\{f_{n}^{\prime \prime}\right\}$ is a sequence $F=\left\{f_{n}\right\}$ of simple $\mathbb{M}$-measurable functions, and by Theorem $4.3, F=E(H, M)$. Finally,

$$
\int_{\mathbf{Q}} \Phi\left(f_{n}-f_{m}\right) d \mu=\int_{\mathbf{Q}_{1}} \Phi\left(f_{n}^{\prime \prime}-f_{m}^{\prime \prime}\right) d \mu_{2} \rightarrow 0
$$

hence $F \in V^{\Phi}(\Omega, \mathcal{U}, \mu, R)$.

For $H \in V_{2}(\Omega, \mathcal{U}, \mu, R)$, the following theorem characterizes $F=E(H, \mathbb{M})$ as the best $V_{2}$-approximation to $H$ by a $L_{2}$-Cauchy sequence of simple $\mathbb{R}$-measurable functions.

Theorem 5.5 (I)arst). If $H \in V_{2}(\Omega, \mathcal{U}, \mu, R)$ and $F=E(H, M)$, then

$$
\begin{aligned}
F & \in V_{2}, \\
\int H K d \mu & \leq \int F K d \mu, \quad K=\left\{k_{m}\right\} \text { M-measurable, } K \in V_{2}, \\
\int H K d \mu & \geq \int F K d \mu, \quad K=\left\{k_{m}\right\} \prod^{c} \text {-measurable, } K \in V_{2}
\end{aligned}
$$

$$
\left(\mathbb{R}^{c}=\left\{A: A^{c} \in \mathbb{R}\right\}\right)
$$




$$
\begin{aligned}
\text { (5.5.4) } \int H F d \mu & =\int F^{2} d \mu, \text { and } \\
\text { (5.5.5) } \int(H-F)^{2} d \mu & \leq \int(H-K)^{2} d \mu, \quad K=\left\{k_{m}\right\} \text { M-measurable, } K \in V_{2} .
\end{aligned}
$$

Moreover, the conditions of the theorem characterize $F$ among the $L_{2}$-Cauchy sequences.

Before giving our proof of Theorem 5.5, we give a geometric interpretation. Brunk [1] used this interpretation to define $f=E\left(b, \mathbb{M}_{2}\right), b \in L_{2}$.

Let $V_{2}(M)$ denote the class of $\mathbb{M}$-measurable $K=\left\{k_{m}\right\}$ in $V_{2}(\Omega, \mathcal{U}, \mu, R)$. If $A\left(V_{2}(\pi)\right)$ is a closed convex cone in a complete inner product space $B\left(V_{2}(\Omega, \mathcal{U}, \mu, R)\right.$ with $\left.(H, F)=\int H F d \mu=\lim _{m \rightarrow \infty}\left(\lim _{n-\infty} \int b_{m} f_{n} d_{\mu}\right)\right)$, and $H \epsilon$ $B$, then there is a unique closest point $F$ of $A$ to $H$, hence (5.5.1) and (5.5.5) and the last assertion of the theorem. Brunk [1, Corollary 2.3, p. 1340] used the notation $F=P(H \mid A)$ ( $P$ for projection) and proved that $F=P(H \mid A)$ if, and only if,

(i) $(H-F, F)=0\left(\int H F d \mu=\int F^{2} d \mu\right)$, i.e., the vector $H-F$ is orthogonal to the vector $F$, and

(ii) $(H-F, K) \leq 0$ for all $K \in A$ ((5.5.2), and also (5.5.3) because if $K$ is $\mathbb{M}^{c}$-measurable, then $-K$ is $\mathbb{M}$-measurable), i.e., the angle between the vector $H-F$ and the vector $K$ is greater than or equal to $90^{\circ}$.

Turning now to the proof of Theorem 5.5, consider the proof of Theorem 5.4 with $\Phi(x)=|x|^{2}$. Then by (5.4.3), $H_{2}(E)=\int_{E} b d \mu_{2}$ for all $E \in \mathcal{U}_{2}$, where $b \epsilon$ $L_{2}\left(\Omega_{1}, \mathcal{U}_{2}, \mu_{2}, R\right)$. Consequently, we can apply Johansen's result [8, Theorem 5, p. 55] for $\sigma$-lattices:

Theorem 5.6 (Johansen). If $b \in L_{1} \cap L_{2}\left(\Omega_{1}, \mathcal{U}_{2}, \mu_{2}, R\right)$, then $f=E\left(b, M_{2}\right)$ is an $\pi_{2}$-measurable function on $\Omega_{1}$ which is characterized by the following conditions:

$$
\begin{aligned}
f & \in L_{1} \cap L_{2} . \\
\int b k d \mu_{2} & \leq \int f k d \mu_{2}, \quad k \mathbb{M}_{2} \text {-measurable, } k \in L_{1} \cap L_{2}, \\
\int b k d \mu_{2} & \geq \int f k d \mu_{2}, \quad k \mathbb{M}_{2}^{c} \text {-measurable, } k \in L_{1} \cap L_{2} . \\
\int b k d \mu_{2} & =\int f^{2} d \mu_{2} .
\end{aligned}
$$

Furthermore,

(5.6.5) $\int(b-f)^{2} d \mu_{2} \leq \int(b-k)^{2} d \mu_{2}, \quad k M_{2}$-measurable, $k \in L_{1} \cap L_{2}$.

With $\Phi(x)=|x|^{2}$, the proof of Theorem 5.4 yields

$$
\int_{\Omega_{1}}\left|b_{n}^{\prime \prime}-b\right|^{2} d \mu_{2} \rightarrow 0, \quad \int_{\Omega_{1}}\left|f_{n}^{\prime \prime}-f\right|^{2} d \mu_{2} \rightarrow 0
$$


and

$$
\int_{\Omega}\left|f_{n}-f_{m}\right|^{2} d \mu=\int_{\Omega_{1}}\left|f_{n}^{\prime \prime}-f_{m}^{\prime \prime}\right|^{2} d \mu_{2} \rightarrow 0 .
$$

Thus, $F=\left\{f_{n}\right\}=E(H, \mathbb{M})$ is in $V_{2}(\Omega, \mathcal{U}, \mu, R)$.

We obtain (5.5.2) and (5.5.3) by applying (5.6.2) and (5.6.3) to

$$
\int H K d \mu=\lim _{n}\left(\lim _{m} \int b_{m} k_{n} d_{\mu}\right)=\lim _{n}\left(\lim _{m} \int b_{m}^{\prime \prime} k_{n}^{\prime \prime} d \mu_{2}\right)=\lim _{n}\left(\int b k_{n}^{\prime \prime} d \mu_{2}\right)
$$

and

$$
\int F K d \mu=\lim _{n}\left(\lim _{m} \int f_{m} k_{n} d_{\mu}\right)=\lim _{n}\left(\lim _{m} \int f_{m}^{\prime \prime} k_{n}^{\prime \prime} d \mu_{2}\right)=\lim _{m}\left(\int f k_{n}^{\prime \prime} d \mu_{2}\right)
$$

respectively.

Since $\int H F d \mu=\int b f d \mu_{2}$ and $\int F^{2} d \mu=\int f^{2} d \mu_{2}$, (5.6.4) implies (5.5.4).

The last property, (5.5.5), now follows from

(A) $\int(H-K)^{2} d \mu=\int(H-F)^{2} d \mu+\int(F-K)^{2} d \mu+2 \int(H-F)(F-K) d \mu \geq 0$

and

$$
\int(H-F)(F-K) d \mu=\int H F d \mu-\int F^{2} d \mu-\int H K d \mu+\int F K d \mu \geq 0 .
$$

Moreover, (A) also shows that if $K$ can play the role of $F$ in the theorem, then $\int(F-K)^{2} d \mu=0$. Hence, the conditions of the theorem characterize the RadonNikodym derivative of $H$ on $M$.

VI. A martingale convergence theorem for lattices. It is, perhaps, appropriate to begin our discussion of martingale convergence with references to some recent work.

In [12], J.J. Uhl discussed convergence in the Orlicz space $L^{\Phi}(\Omega, \mathscr{Q}, \mu, B)$ ( $\mathfrak{A}$ a $\sigma$-algebra and $\mu \sigma$-additive) of Banach space valued martingales $\left\{f_{t}, \mathbb{Q}_{t}\right\}$, where $\left\{\mathbb{Q}_{t}\right\}$ is an increasing net of sub- $\sigma$-algebras and $f_{t_{1}}=E\left(f_{t_{2}}, \mathbb{Q}_{t_{1}}\right)$ for $t_{2} \geq$ $t_{1}$. In [13], Uhl discussed convergence in the Orlicz space $V^{\Phi}(\Omega, \mathscr{Q}, \mu, B)(\mathscr{Q}$ an algebra and $\mu$ finitely additive) of martingales $\left\{F_{t}, Q_{t}\right\}$ of Banach space valued additive set functions $F_{t}$, where $\left\{\mathscr{Q}_{t}\right\}$ is an increasing net of subalgebras and $F_{t_{1}}=E\left(F_{t_{2}}, \mathbb{Q}_{t_{1}}\right)$ for $t_{2} \geq t_{1}$. Uhl [13] noted that some of the results he obtained for additive set functions defined on algebras of sets can be deduced from known results for $\sigma$-additive set functions defined on $\sigma$-algebras of sets by using the isomorphisms $\tau, T$, and $U$.

H. D. Brunk and S. Johansen [2, Theorem 2.8, p. 605] proved an almost everywhere martingale convergence theorem for the Radon-Nikodym derivatives given an increasing sequence of $\sigma$-lattices. Norm convergence (in the Orlicz space 
$L^{\Phi}(\Omega, \mathbb{A}, \mu, R), \mathbb{Q}$ a $\sigma$-algebra and $\mu \sigma$-additive $)$ of these derivatives was established in [4, Theorem 2].

In [3], R. B. Darst established which features of a martingale convergence theorem carry over to the setting of additive set functions defined on lattices of sets: proving convergence in $V_{1}(\Omega, \mathfrak{Q}, \mu, R)$ of martingales of Radon-Nikodym derivatives given a lattice (Definition 4.4). Using the isomorphisms $\tau, T$, and $U$ and applying the corresponding martingale convergence theorem for $\sigma$-lattices, [4, Theorem 2], we are able to obtain an extension of Darst's result to $V^{\boldsymbol{\Phi}}(\Omega, \mathcal{U}, \mu, R)$. We begin by establishing the necessary notation.

Suppose that $\{M(k)\}$ is an increasing sequence of lattices of subsets of $\Omega$, and $\mathbb{M}=\bigcup_{k} \mathbb{M}(k)$. Then the algebras $\mathbb{Q}(k)$ of subsets of $\Omega$ that are generated by these lattices increase to the algebra $\mathfrak{Q}$ generated by the lattice $\mathbb{M}$.

Suppose that $\lambda$ and $\mu$ are nonnegative bounded additive functions on $\mathscr{Q}$, and that $\lambda \in V^{\Phi}(\Omega, \mathbb{Q}, \mu, R)$. Denote by $\lambda(k)$ and $\mu(k)$ the restrictions of $\lambda$ and $\mu$ to $\mathfrak{A}(k)$. Then $\lambda(k)$ is in $V^{\Phi}(\Omega, \mathfrak{A}(k), \mu(k), R)$. Let $E(\lambda, \mathbb{M})=F=\left\{f_{n}\right\}_{n=1}^{\infty}$ and $E(\lambda(k), \mathbb{M}(k))=G_{k}=\left\{g_{k, n}\right\}_{n=1}^{\infty}$. By Theorem 5.4, $F$ and $G_{k}$ are in $V^{\Phi}$.

Because the sequences $\left\{g_{k, n}\right\}_{n=1}^{\infty}$ are Cauchy sequences in $L^{\Phi}(\Omega, \mathbb{Q}(k), \mu(k), R)$, they are Cauchy sequences in $L^{\Phi}(\Omega, \mathbb{Q}, \mu, R)$ (i.e. $\left.\int_{\Omega} \Phi\left(g_{k, n}-g_{k, m}\right) d \mu=\int_{\Omega} \Phi\left(g_{k, n}-g_{k, m}\right) d \mu(k)\right)$. Hence the equation

$$
H_{k}(E)=\lim _{n \rightarrow \infty} \int_{E} g_{k, n} d \mu, \text { for } E \in \mathbb{P},
$$

defines an additive extension of $G_{k}$ to $\mathscr{Q}$. Notice that $\left\{H_{k}\right\}$ is determined by $\{\Re(k)\}, \lambda$, and $\mu$. We call $\left\{H_{k}, \mathbb{N}(k)\right\}$ a martingale, and prove the following martingale convergence theorem.

Theorem 6.1. The sequence $\left\{H_{k}\right\}$ converges to $F$ in $V^{\Phi}(\Omega, Q, \mu, R)$.

Remark. By assuming $\lambda \in V^{\Phi}$, we are assuming $\lambda$ is absolutely continuous with respect to $\mu$. Note that $\lambda$ is absolutely continuous with respect to $\mu$ if, and only if, the $\lambda(k)$ 's are uniformly absolutely continuous with respect to the $\mu(k)$ 's (i.e. for each $\epsilon>0$ there is a $\delta>0$ such that if $E \in \mathbb{P}(k)$ and $\mu(k)(E)<\delta$, then $\lambda(k)(E)<\epsilon)$. An example illustrating this fact and which also shows that the requirement that $\lambda$ be absolutely continuous with respect to $\mu$ is not superfluous can be found in [3, p. 597].

Proceeding to the proof of the theorem, apply the isomorphisms $\tau, T$, and $U$ to obtain

$$
\begin{aligned}
\mathbb{P} \rightarrow \mathbb{P}_{1} \rightarrow \mathbb{P}_{2}, & \mathbb{N}(k) \rightarrow \mathbb{M}(k, 1) \rightarrow \mathbb{M}(k, 2), \\
\mathbb{N} \rightarrow \mathbb{M}_{1} \rightarrow \mathbb{M}_{2}, & \lambda \rightarrow \lambda_{1} \rightarrow \lambda_{2}, \\
\mathbb{P}(k) \rightarrow \mathbb{P}(k, 1) \rightarrow \mathbb{P}(k, 2), & \mu \rightarrow \mu_{1} \rightarrow \mu_{2} .
\end{aligned}
$$


Then define $\lambda(k, i)=\lambda_{i} \mid \mathfrak{Q}(k, i)$ and $\mu(k, i)=\mu_{i} \mid \mathfrak{Q}(k, i)$ for $i=1,2$.

Appealing to the proof of Theorem 5.4, in particular (5.4.3), we may assume that

$$
\lambda_{2}(E)=\int_{E} b d \mu_{2}, \quad b \in L^{\Phi}\left(\Omega_{1}, \mathbb{P}_{2}, \mu_{2}, R\right),
$$

and

$$
\lambda(k, 2)(E)=\int_{E} b_{k} d \mu_{2}, \quad b_{k} \in L^{\Phi}\left(\Omega_{1}, \mathbb{P}(k, 2), \mu(k, 2), R\right) .
$$

Setting $f=E\left(b, M_{2}\right)$ and $d_{k}=E\left(b_{k}, M(k, 2)\right)$, it follows from the corresponding martingale convergence theorem for $\sigma$-lattices, [4, Theorem 2], that

$$
\lim _{k \rightarrow \infty} \int_{\Omega_{1}} \Phi\left(f-d_{k}\right) d \mu_{2}=0
$$

Recall (see discussion preceding Theorem 4.3) that the sequences $\left\{g_{k, n}\right\}=H_{k}$ and $\left\{f_{n}\right\}=F$ are the inverse images under $\tau$ of sequences $\left\{g_{k, n}^{\prime \prime}\right\}$ and $\left\{f_{n}^{\prime \prime}\right\}$ such that

$$
\begin{gathered}
\lim _{n \rightarrow \infty} \int_{\Omega_{1}} \Phi\left(d_{k}-g_{k, n}^{\prime \prime}\right) d \mu_{2}=0, \text { and } \\
\lim _{n \rightarrow \infty} \int_{\Omega_{1}} \Phi\left(f-f_{n}^{\prime \prime}\right) d \mu_{2}=0 .
\end{gathered}
$$

Thus,

$$
\left\|H_{k}-F\right\|_{\Phi}=\lim _{n \rightarrow \infty}\left\|g_{k, n}-f_{n}\right\|_{\Phi}=\lim _{n \rightarrow \infty}\left\|g_{k, n}^{\prime \prime}-f_{n}^{\prime \prime}\right\|_{\Phi}=\left\|d_{k}-f\right\|_{\Phi}
$$

which completes the proof of Theorem 6.1.

Recalling [14] that $\lambda \in V^{\Phi}(\Omega, \mathbb{A}, \mu, R)$ if, and only if, $I_{\Phi}(\lambda)<\infty$ and $\lambda$ is $\mu$-continuous, and that $\lambda$ is $\mu$-continuous if, and only if, the $\lambda(k)$ 's are uniformly absolutely continuous with respect to the $\mu(k)$ 's, we obtain the following corollary.

Corollary 6.2. Suppose that $\mathbb{M}(k)$ is an increasing sequence of lattices of subsets of $\Omega$. For each positive integer $k$, suppose each of $\lambda(k)$ and $\mu(k)$ is a nonnegative additive set function defined on $\mathbb{Q}(k)$, the algebra generated by $\mathbb{M}_{k}$, such that $\lambda(k+1)|\mathfrak{Q}(k)=\lambda(k), \mu(k+1)| \mathfrak{Q}(k)=\mu(k), \lambda(k) \in V^{\Phi}(\Omega, \mathbb{P}(k), \mu(k), R)$, and $I_{\Phi}(\lambda(k)) \leq M$. If $\mathbb{A}=\bigcup_{k=1}^{\infty} \mathfrak{A}(k)$, then $\lambda(k)$ and $\mu(k)$ are in $V^{\Phi}(\Omega, \mathbb{P}, \mu, R)$, where $\mu(E)=\mu(k)(E)$ if $E$ is in $\mathbb{Q}(k)$. Finally, suppose that the $\lambda(k)$ 's are uniformly absolutely continuous with respect to the $\mu(k)$ 's. Then the sequence $\left\{H_{k}\right\}$ converges in norm.

Note. With $\Phi(x)=|x|$ (i.e. Darst's result) the requirement that $I_{\Phi}(\lambda(k)) \leq M$ is superfluous because in this case $\lambda$ absolutely continuous with respect to $\mu$ 
implies that $\lambda$ is of bounded variation (cf. [10, Theorem 1, p. 529]).

VII. Vector valued set functions. In this section we use Definition 4.2 coordinatewise to define the Radon-Nikodym derivative of a bounded additive vector valued set function, $\lambda: \mathbb{M} \rightarrow R^{n}$, with respect to a nonnegative bounded additive set function, $\mu: \mathbb{M} \rightarrow R$, on a lattice $\mathbb{M}$ of subsets of a nonempty set $\Omega$. Then the "best approximation" theorem, Theorem 5.5, and the martingale convergence theorem, Theorem 6.1, are extended to this case. We begin by introducing some notation.

If $x \in R^{n}$, then $x=\sum_{i=1}^{n} x_{i} e_{i}$, where $e_{i}=\left(e_{i 1}, \cdots, e_{i n}\right)$, with $e_{i j}=0$ for $i \neq j$ and $e_{i j}=1$ for $i=j$. We will use the norm (recall that all norms on $R^{n}$ are equivalent)

$$
\|x\|=\sum_{i=1}^{n}\left|x_{i}\right| .
$$

We partially order $R^{n}$ by the order relation " $\leq$ ", where $x \leq y$ if $x_{i} \leq y_{i}$, $i=$ $1, \cdots, n$. Then $|x|=\sup (x,-x)=\sum_{i=1}^{n}\left|x_{i}\right| e_{i}$ and $|x| \leq|y| \rightrightarrows\|x\| \leq\|y\|$ (i.e., $R^{n}$ with this ordering is a Banach lattice).

If $f: \Omega \rightarrow R^{n}$, then $f(x)=\sum_{i=1}^{n} f_{i}(x) e_{i}$, where $f_{i}: \Omega \rightarrow R$.

Definition 7.1. A function $f: \Omega \rightarrow R^{n}$ is said to be $\mathbb{M}$-measurable if each coordinate function $f_{i}: \Omega \rightarrow R, i=1, \cdots, n$, is M-measurable (Definition 2.3); i.e., for all $a \in R^{n},\left[f_{i}>a_{i}\right] \in M, i=1, \cdots, n$.

Let $P^{n}=\left\{x \in R^{n}: \theta \leq x\right.$, where $\left.\theta=(0, \cdots, 0)\right\}$ and suppose that $\lambda: \mathbb{M} \rightarrow$ $P^{n}$ and $\mu: \mathbb{M} \rightarrow P^{1}$ are bounded additive (Definition 2.2) set functions. The unique extensions [11, Corollary 1.2.1, p. 189] of $\lambda$ and $\mu$ to the algebra $\mathcal{P}$, generated by the lattice $M$, are also denoted by $\lambda$ and $\mu$. Then

$$
\lambda(E)=\sum_{i=1}^{n} \lambda_{i}(E) e_{i}, \quad E \in \mathbb{T},
$$

where $\lambda_{i}: \mathfrak{Q} \rightarrow P^{1}$ is a bounded additive function on $\mathbb{Q}, i=1, \ldots, n$.

Definition 7.2. The Radon-Nikodym derivative of $\lambda$ with respect to $\mu$ on $\mathbb{R}$ is a $L_{1}$-Cauchy sequence $F=\left\{f_{k}\right\}_{k=1}^{\infty}$ of simple $\mathbb{N}$-measurable vector valued functions, where $f_{k}(x)=\sum_{i=1}^{n} f_{i, k}(x) e_{i}$ and $F_{i}=\left\{f_{i, k}\right\}_{k=1}^{\infty}$ is the derivative of $\lambda_{i}$ with respect to $\mu$ on $\mathbb{M}$ (Definition 4.2). Thus $F(E)=\sum_{i=1}^{n} F_{i}(E) e_{i}, E \in \mathbb{Q}$.

The sequence $F=\left\{f_{k}\right\}_{k=1}^{\infty}$ is a $L_{1}$-Cauchy sequence because

$$
\int_{\Omega}\left\|f_{N}(x)-f_{M}(x)\right\| d \mu=\sum_{i=1}^{n} \int_{\Omega}\left|f_{i, N}(x)-f_{i, M}(x)\right| d \mu \rightarrow 0 .
$$

Thus $F \in V_{1}\left(\Omega, \mathbb{Q}, \mu, R^{n}\right)$.

Suppose now that $\mathcal{U}$ is an algebra of subsets of $\Omega$, that $\pi \subset \mathcal{U}$, and that $\mu: \mathcal{U} \rightarrow P^{1}$ is a bounded additive function. Recall that $H \in V^{\Phi}\left(\Omega, \mathcal{U}, \mu, R^{n}\right)$ can be identified with a sequence $\left\{b_{k}\right\}$ of simple $\mathcal{U}$-measurable vector valued functions 
such that $\int_{\Omega} \Phi\left(\left\|b_{k}(x)-b_{m}(x)\right\|\right) d \mu \rightarrow 0$ (as $\left.k, m \rightarrow \infty\right)$.

Definition 7.3. If $\lambda$ is the restriction to $\mathbb{N}$ of $H \in V^{\Phi}\left(\Omega, \mathcal{U}, \mu, R^{n}\right)$, then we denote the derivative, $F=\left\{f_{k}\right\}_{k=1}^{\infty}$, of $\lambda$ with respect to $\mu$ on $\mathbb{M}$ by $F=E(H, M)$.

If $H \in V^{\Phi}\left(\Omega, \mathcal{U}, \mu, R^{n}\right)$, then $H(E)=\Sigma_{i=1}^{n} H_{i}(E) e_{i}$, where $H_{i} \in V^{\Phi}(\Omega, \mathcal{U}, \mu, R)$. Thus

$$
F(A)=E(H, \mathbb{M})(A)=\sum_{i=1}^{n} E\left(H_{i}, \mathbb{M}\right)(A) e_{i}=\sum_{i=1}^{n} F_{i}(A) e_{i}, \quad A \in \mathcal{U} .
$$

The sequence $F=\left\{f_{k}\right\}_{k=1}^{\infty}$ is a $L^{\Phi}$-Cauchy sequence because the convexity of $\Phi$ and the $\Delta_{2}$-condition imply that

$$
\int_{\Omega} \Phi\left(\left\|f_{N}(x)-f_{M}(x)\right\|\right) d \mu \leq C \sum_{i=1}^{n} \int_{\Omega} \Phi\left(\left|f_{i, N}(x)-f_{i, M}(x)\right|\right) d \mu \rightarrow 0 .
$$

Hence $F \in V^{\Phi}\left(\Omega, \mathcal{U}, \mu, R^{n}\right)$, i.e., the derivative of a $V^{\Phi}$-function is a $V^{\Phi}$-function.

Theorem 7.4. The derivative of a function $H \in V^{\Phi}\left(\Omega, \mathcal{U}, \mu, R^{n}\right)$ is a function $F=E(H, \mathbb{M}) \in V^{\Phi}\left(\Omega, \mathcal{U}, \mu, R^{n}\right)$.

That $F=E(H, \mathbb{M})$ is the best $V_{2}$-approximation to $H \in V_{2}\left(\Omega, \mathcal{U}, \mu, R^{n}\right)$ by a $L_{2}$-Cauchy sequence of simple $\mathbb{M}$-measurable functions follows immediately from Theorem 5.5.

Theorem 7.5. If $H \in V_{2}\left(\Omega, \mathcal{U}, \mu, R^{n}\right)$ and $F=E(H, M)$, then

$$
\begin{aligned}
& F \in V_{2}, \\
& \int H K d \mu \leq \int F K d \mu, \quad K \mathbb{M}_{\text {-measurable, } K \in V_{2},} \\
& \int H K d \mu \geq \int F K d \mu, \quad K \mathbb{M}^{c} \text {-measurable, } K \in V_{2}, \\
& \int H F d \mu=\int F^{2} d \mu, \\
& \int(H-F)^{2} d \mu \leq \int(H-K)^{2} d \mu, \quad K \text { M-measurable, } K \in V_{2} .
\end{aligned}
$$

Moreover, the conditions of the theorem characterize $F$ among the $L_{2}$-Cauchy sequences.

Proof. We simply apply Theorem 5.5 to each coordinate. For example,

$$
\int H K d \mu=\sum_{i=1}^{n} \int H_{i} K_{i} d \mu \leq \sum_{i=1}^{n} \int F_{i} K_{i} d \mu=\int F K d \mu .
$$

Since $\|H\|_{\Phi}=\left\|\Sigma_{i=1}^{n} H_{i} e_{i}\right\|_{\Phi} \leq \Sigma_{i=1}^{n}\left\|H_{i}\right\|_{\Phi}$ for $H \in V^{\Phi}\left(\Omega, U, \mu, R^{n}\right)$, we can obtain a martingale convergence theorem by applying Theorem 5.1 coordinatewise. To this end, we first establish the necessary notation. 
Suppose that $\left\{\pi_{k}\right\}$ is an increasing sequence of lattices of subsets of $\Omega$, and $M=\bigcup_{k} \pi_{k}$. Then the algebras $\mathbb{A}_{k}$ of subsets of $\Omega$ that are generated by these lattices increase to the algebra $\mathfrak{Q}$ generated by the lattice $\mathbb{M}$.

Suppose that $\lambda: \mathbb{A} \rightarrow P^{n}$ and $\mu: \mathbb{Q} \rightarrow \dot{P}^{1}$ are bounded additive functions on $\mathfrak{Q}$, and that $\lambda \in V^{\Phi}\left(\Omega, \mathfrak{Q}, \mu, R^{n}\right)$. Denote by $\lambda^{k}$ and $\mu_{k}$ the restrictions of $\lambda$ and $\mu$ to $\mathfrak{Q}_{k}$. Then $\lambda^{k}$ is in $V^{\Phi}\left(\Omega, \mathbb{C}_{k}, \mu_{k}, R^{n}\right)$. Let $E(\lambda, \mathbb{M})=F=\left\{f_{m}\right\}_{m=1}^{\infty}$ and $E\left(\lambda^{k}, \Re_{k}\right)=G^{k}=\left\{g_{k, m}\right\}_{m=1}^{\infty}$. By Theorem 7.4,F and $G^{k}$ are in $V^{\Phi}$.

Because the sequences $G^{k}=\left\{g_{k, m}\right\}_{m=1}^{\infty}$ are Cauchy sequences in $L^{\Phi}\left(\Omega, \mathbb{A}_{k}, \mu_{k}, R^{n}\right)$ they are Cauchy sequences in $L^{\Phi}\left(\Omega, \mathbb{Q}, \mu, R^{n}\right)$ (i.e. $\int_{\Omega} \Phi\left(\left\|g_{k, l}(x)-g_{k, m}(x)\right\| d \mu=\int_{\Omega} \Phi\left(\left\|g_{k, l}(x)-g_{k, m}(x)\right\|\right) d \mu_{k}\right)$. Hence the equation

$$
H^{k}(E)=\lim _{m \rightarrow \infty} \int_{E} g_{k, m} d \mu, \quad \text { for } E \in \mathbb{Q},
$$

defines an additive extension of $G^{k}$ to $\uparrow$. Notice that $\left\{H^{k}\right\}$ is determined by $\left\{\mathbb{M}_{k}\right\}, \lambda$, and $\mu$. We call $\left\{H^{k}, \mathbb{M}_{k}\right\}$ a martingale.

Theorem 7.6. The sequence $\left\{H^{k}\right\}$ converges to $F$ in $V^{\Phi}\left(\Omega, \mathfrak{Q}, \mu, R^{n}\right)$.

Proof. Suppose $\lambda(E)=\sum_{i=1}^{n} \lambda_{i}(E) e_{i}$, then $\lambda^{k}(E)=\sum_{i=1}^{n} \lambda_{i}^{k}(E) e_{i}$, where $\lambda_{i}^{k}$ is the restriction of $\lambda_{i}$ to $\mathscr{\mathbb { A }}_{k}$. Set $F_{i}=E\left(\lambda_{i}, \mathbb{M}\right), G_{i}^{k}=E\left(\lambda_{i}^{k}, \mathbb{M}_{k}\right)=$ $\left\{g_{i, k, m}\right\}_{m=1}^{\infty}$, and

$$
H_{i}^{k}(E)=\lim _{m \rightarrow \infty} \int_{E} g_{i, k, m} d \mu, \quad E \in \mathbb{P} .
$$

Then by Theorem 6.1 , for $i=1, \ldots, n$,

$$
\left\|H_{i}^{k}-F_{i}\right\|_{\Phi} \rightarrow 0(\text { as } k \rightarrow \infty) \text {. }
$$

Finally, since $H^{k}(E)=\sum_{i=1}^{n} H_{i}^{k}(E) e_{i}$ and $F(E)=\sum_{i=1}^{n} F_{i}(E) e_{i^{\prime}}$

$$
\left\|H^{k}-F\right\|_{\Phi} \leq \sum_{i=1}^{n}\left\|H_{i}^{k}-F_{i}\right\|_{\Phi} \rightarrow 0(\text { as } k \rightarrow \infty) \text {. }
$$

\section{BIBLIOGRAPHY}

1. H. D. Brunk, Conditional expectation given a $\sigma$-lattice and applications, Ann. Math. Statist. 36 (1965), 1339 - 1350. MR 32 \#3091.

2. H. D. Brunk and S. Johansen, A generalized Radón-Nikodym derivative, Pacific J. Math. 34 (1970), 585-617. MR 42 \# 1968.

3. R. B. Darst, The Lebesgue decomposition, Radón-Nikodym derivative, conditional expectation, and martingale convergence for lattices of sets, Pacific J. Math. 35 (1970), $581-600$.

4. R. B. Darst and Gene A. De Both, Norm convergence of martingales of Radón-Nikodym derivatives given a $\sigma$-lattice, Pacific J. Math. (to appear).

5. - Two approximation properties and a Radón Nikodym derivative for lattices of sets (to appear). 
6. N. Dunford and J. T. Schwartz, Linear operators. I: General theory, Pure and Appl. Math., vol. 7, Interscience, New York, 1958. MR 22 \#3302.

7. P. R. Halmos, Measure theory, Van Nostrand, Princeton, N.J., 1950. MR 11, 504.

8. S. Johansen, The descriptive approach to the derivative of a set function with respect to a $\sigma$-lattice, Pacific J. Math. 21 (1967), 49-58.

9. M. A. Krasnosel' skii and Ja. B. Rutickir., Convex functions and Orlicz spaces, GITTL, Moscow, 1958; English transl., Noordhoff, Groningen, 1961. MR 21 \#5144; MR 23 \# A4016.

10. S. Leader, The theory of $L^{p}$-spaces for finitely additive set functions, Ann. of Math. (2) 58 (1953), 528-543. MR 15, 326.

11. B. J. Pettis, On the extension of measures, Ann. of Math. (2) 54 (1951), 186197. MR 13, 19.

12. J. J. Uhl, Jr., Applications of Radón-Nikodym theorems to martingale convergence, Trans. Amer. Math. Soc. 145 (1969), 271 - 285. MR 40 \# 4983.

13. - Martingales of vector valued set functions, Pacific J. Math 30 (1969), 533-548. MR $40 \# 1767$.

14. - Orlicz spaces of finitely additive set functions, Studia Math. 29 (1967), 19-58. MR $37 \# 1985$.

DEPARTMENT OF MATHEMATICS, ST. NORBERT COLLEGE, DE PERE, WISCONSIN 54115 